\title{
Echocardiographic Diagnosis of Aortic Intramural Hematoma via the Posterior Paraspinal Window
}

\author{
Tsuyoshi Yoshimuta ${ }^{1}$, Hiroyuki Yokoyama ${ }^{1}$, Toshiya Okajima ${ }^{1}$, Masakazu Yamagishi ${ }^{2}$ \\ and Hiroshi Nonogi ${ }^{1}$
}

(Inter Med 49: 83-84, 2010)

(DOI: 10.2169/internalmedicine.49.2838)
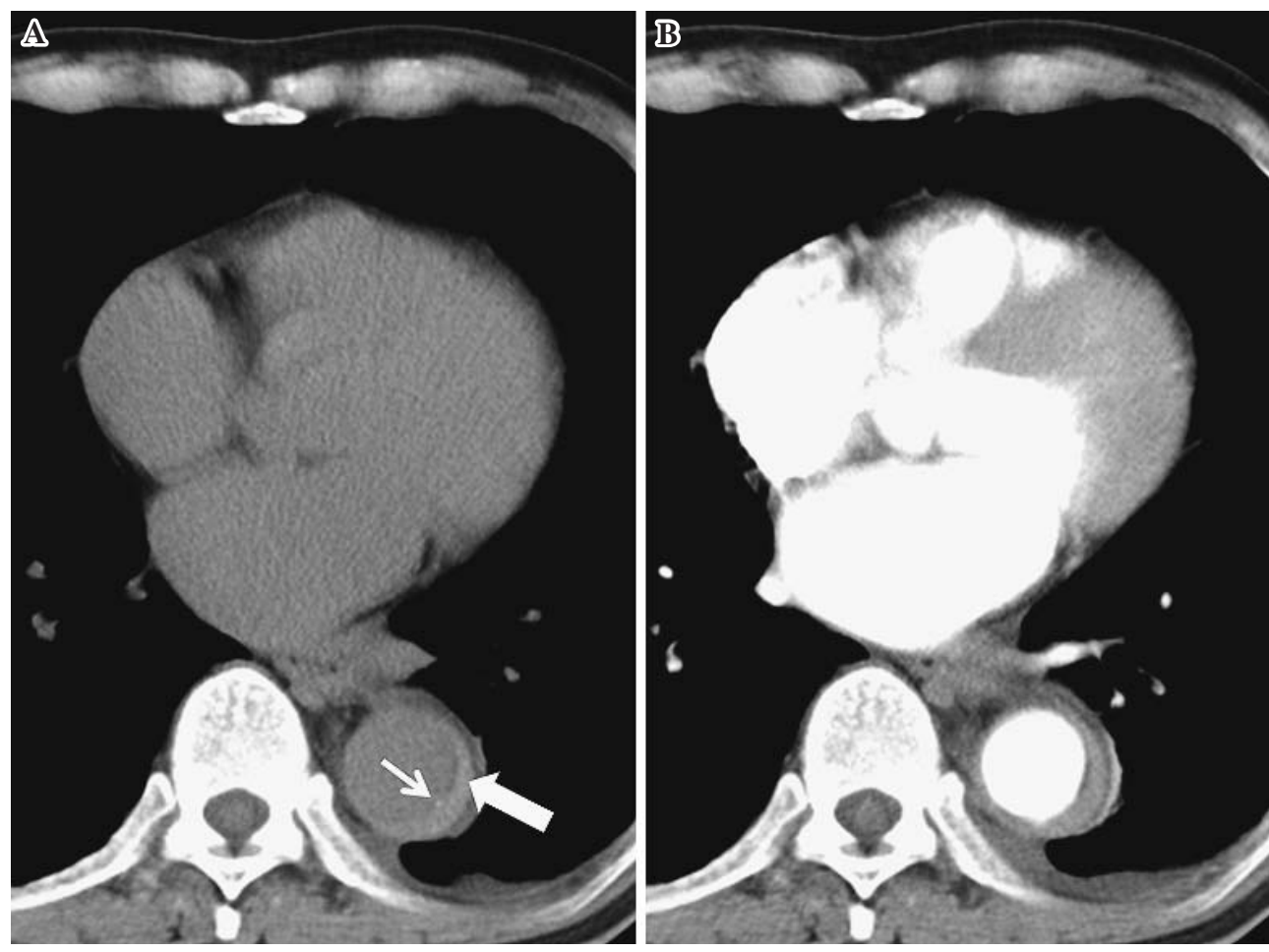

Picture 1. Non-contrast-enhanced axial CT scan shows crescentic thickening and high attenuation of the aortic wall from the 1 o'clock to the 8 o'clock position (large arrow), as well as displacement of intimal calcification (small arrow) in the descending thoracic aorta (A). Contrast-enhanced axial CT scan at the same level shows that there is no intimal flap and a communication within the area of thickening (B).

A 72-year-old man was referred to our hospital with severe back pain lasting for 2 days. Computed tomography (CT) showed crescentic thickening and high attenuation areas along the aortic wall extending from the proximal descending thoracic aorta to the origin of the celiac artery from the abdominal aorta, which represented intramural he- matoma (IMH) in the descending thoracic aorta (Picture 1). After CT scanning, the patient underwent echocardiography with a Prosound $\alpha 7$ (Aloka, Tokyo, Japan) in the left lateral decubitus position using a sector transducer. Transthoracic echocardiography via the parasternal, apical, and subcostal windows did not demonstrate IMH. When the trans-

${ }^{1}$ Department of Medicine (Cardiology), National Cardiovascular Center, Osaka and ${ }^{2}$ Division of Cardiovascular Medicine, Kanazawa University Graduate School of Medical Science, Kanazawa

Received for publication September 1, 2009; Accepted for publication September 16, 2009

Correspondence to Dr. Tsuyoshi Yoshimuta, tyoshimu@hsp.ncvc.go.jp 

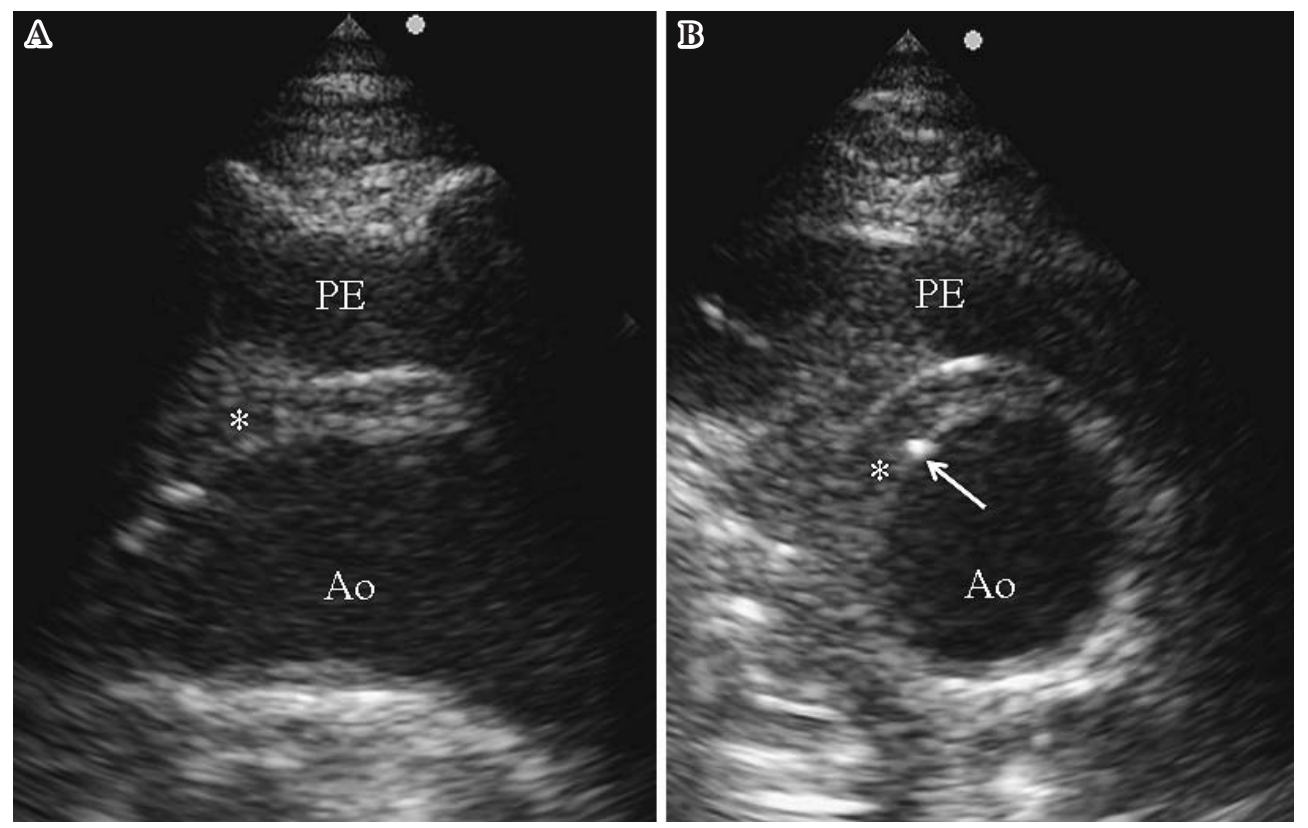

Picture 2. Posterior paraspinal long-axis (A) and short-axis (B) views of the descending thoracic aorta show crescentic aortic wall thickening, a thrombus-like appearance, and central displacement of intimal calcification (white arrows), findings that indicate the presence of an IMH (*). Ao, aorta; PE, pleural effusion.

ducer was positioned to the left of the spine for the posterior paraspinal window $(\mathrm{PPW})$, crescentic thickening of the aortic wall clearly demonstrated that it was approximately 8 $\mathrm{mm}$ in size and had a thrombus-like appearance; moreover central displacement of intimal calcification was also observed (Picture 2). Thus, echocardiography via the PPW facilitated detection of the CT findings.

IMH and acute aortic dissection (AAD) need to be diagnosed rapidly due to the high risk of early death. CT scanning and transesophageal echocardiography are the most useful imaging modalities for diagnosing IMH and AAD. However, it is not easy to perform these examinations immediately at bedside. In contrast, echocardiography via the
PPW enables immediate, noninvasive, and easy evaluation of patients for suspected IMH or AAD at bedside, even in the presence of a pleural effusion. To our knowledge, there has only been one prior report, in which Klein et al showed that the PPW was useful to diagnose aortic dissection with true and false lumens separated by an intimal flap (1). However, there have not been any reports about the evaluation of IMH via the PPW. As demonstrated in this case, echocardiography via the PPW makes it possible to diagnose IMH. Echocardiography via the PPW generates CT-like images and appears to be useful for the noninvasive diagnosis of IMH of the descending thoracic aorta.

\section{Reference}

1. Klein AL, Chan KL, Walley V. A new paraspinal window in the echocardiographic diagnosis of descending aortic dissection. Am
Heart J 114: 902-904, 1987.

(C) 2010 The Japanese Society of Internal Medicine http://www.naika.or.jp/imindex.html 